\title{
The Design of Follow-up Hydraulic Muffler Based on Spring-Damp Regulating Device
}

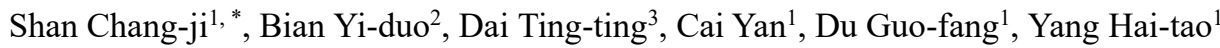 \\ ${ }^{1}$ Institute of Physics and Information Engineering, Zhaotong University, Zhaotong,657000, PRC \\ ${ }^{2}$ School of Foreign Languages, Zhaotong University, Zhaotong, 657000, Yunnan, PRC \\ ${ }^{3}$ School of Mathematics and Statistics, Zhaotong University, Zhaotong, 657000, Yunnan, PRC
}

\begin{abstract}
The follow-up hydraulic muffler can make the natural frequency of the muffler equal to systematic pulsation frequency in time by changing the section area of the quality chamber so as to achieve the best attenuation effect. In this paper, a spring-damp regulator is installed on the basis of the hydraulic muffler, and the static and dynamic characteristics of the follow-up hydraulic muffler are analyzed by measuring the rotational speed of the pump with the sensor. The results show that the hydraulic muffler based on the spring-damp regulator can effectively attenuate the pulsation.
\end{abstract}

\section{Introduction}

In the system using fluid transfer power, the output of the power source is constant. However, the load and hydraulic system change themselves. The pump speed is closely related to the working conditions, and thus the natural frequency of the system changes as the rotational speed changes. There are flow pulsation and pressure pulsation in the hydraulic pipeline and the existence of pulsation produces noise, which directly affects the efficiency and service life of the system. Therefore, how to reduce the flow pulsation becomes an important issue.

The hydraulic muffler is a kind of muffler which is designed according to the working principle of air muffler and the physical characteristics of working medium of hydraulic system.It is actually used to attenuate and absorb hydraulic pulsation, so it is also called hydraulic attenuator or hydraulic filter.Mufflers are mainly divided into two categories: resistive muffler and resistance muffler.Resistive hydraulic attenuator is seldom used in hydraulic system because of its complex structure, large pressure loss and short service life.However, resistance hydraulic muffler is widely used because of its simple structure, small pressure loss and long service life.

The hydraulic muffler is a structural parameter element,which mainly consist of quality chamber and volume chamber, meanwhile, it is mainly designed for a specific frequency attenuation.If the vibration frequency of the system deviates from a specific frequency, the attenuation effect of the hydraulic muffler will be greatly reduced.Therefore, the design of follow-up hydraulic muffler has become a new research direction.

\section{The Structural Design of Follow-up Hydraulic Muffler}

The working principle of the follow-up hydraulic muffler is that the structure parameters of the muffler change with the change of the system pulsation frequency,making the natural frequency of the muffler equal to the systematic pulsation frequency,so as to achieve the purpose of best pulsation attenuation.Reference [1] shows that with the increase of the length of the chamber, the peak frequency and the amplitude of pulsation attenuation of the absorbed noise source change little.If it moves slightly to the low frequency, the change of length of the volume chamber has little effect on the amplitude of pulsation attenuation. Yet with the increase of the aperture of the quality chamber, the peak frequency of the absorption noise source moves rapidly to high frequency,and the pulse attenuation increases rapidly.Therefore, this paper chooses the way of changing the aperture of the quality chamber to design the follow-up hydraulic muffler.The structural design is shown in Fig.1.

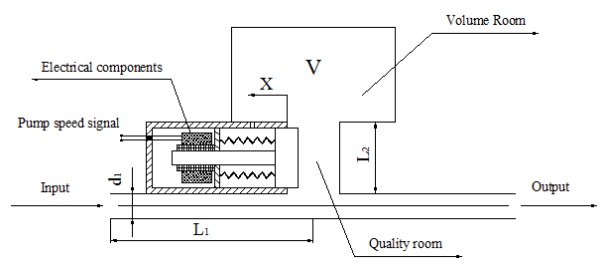

Fig.1. The structural design of the follow-up hydraulic muffler with spring-damp regulator

The working principle of the follow-up hydraulic muffler with spring-damp regulator is as follows: when the rotational speed of hydraulic pump increases, the

* Corresponding author: shanchangji@126.com 
rotational speed signal measured by the speed sensor increases, and the signal is input to the electrical component to form the signal current.The signal current is gradually amplified by the current amplifier and the control amplifier to form the control current.The control current is transferred to the electromagnet, which produces magnetic attraction. This magnetic attraction makes the sliding block moves toward $X$ direction, the larger the aperture of the quality chamber, the higher the natural resonance frequency and the main anti-resonance frequency of the follow-up hydraulic muffler. When the magnetic attraction of the electromagnet is balanced with the spring force,the sliding block will stop moving and the aperture of the quality chamber keeps stable ${ }^{[2]}$.Under this aperture condition, the natural resonance frequency or the main anti-resonance frequency of the follow-up hydraulic muffler is equal to the natural frequency or the pulsation frequency of the hydraulic pump, thus attenuating the amplitude of pulsation to a large extent. When the rotational speed of the hydraulic pump decreases, the rotational speed signal measured by the speed sensor decreases, and the magnetic attraction decreases due to the smaller control current. Under the dominant action of the spring force, the sliding block moves toward the $-X$ direction. When the magnetic attraction equals the spring force, the sliding block stops moving and the aperture of the quality chamber changes again,so that the natural resonance frequency or the main anti-resonance frequency of the follow-up hydraulic

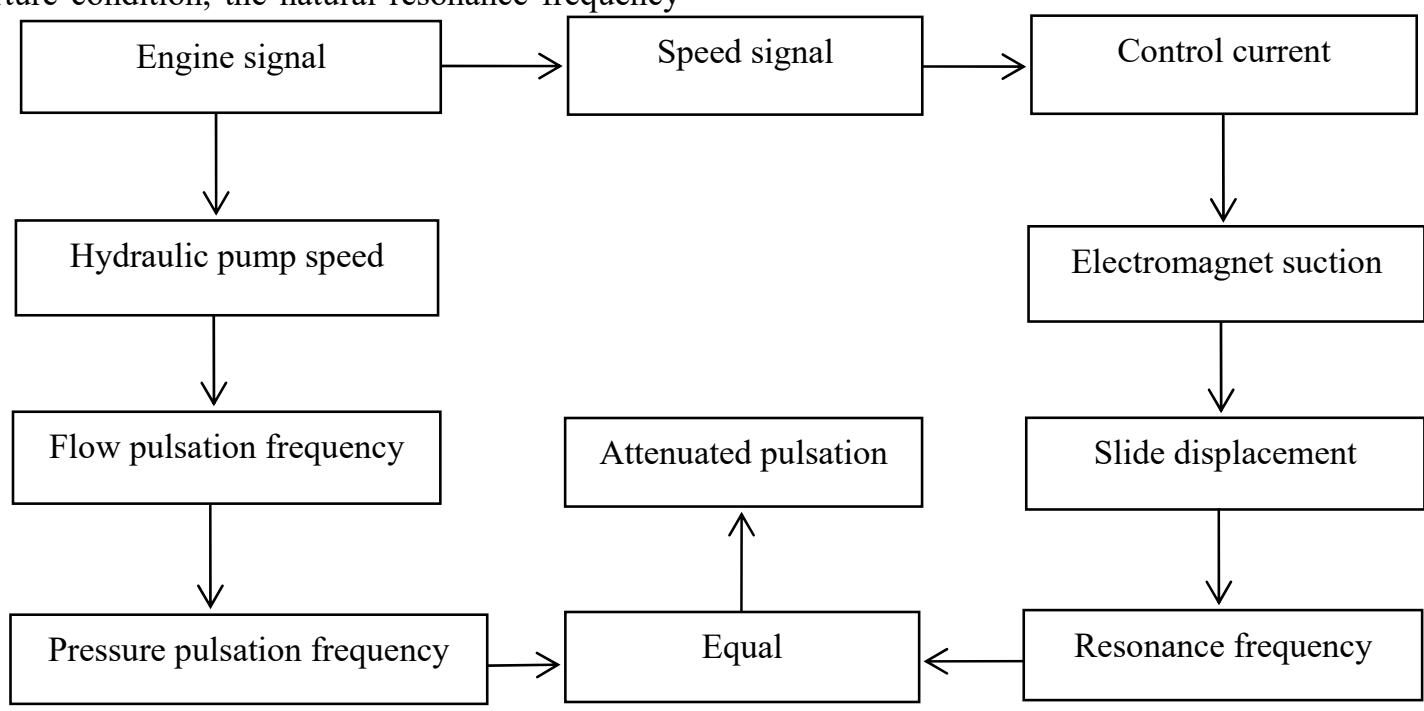

Fig. 2. The diagram of the working principle of a follow-up hydraulic muffler

muffler equals the natural frequency or the pulsation frequency of the hydraulic pump.According to the above working principle, the follow-up hydraulic muffler can respond to the change of the natural frequency of the system in time,so that the attenuation effect can be maintained at a good level to meet the design target.Fig. 2 is the diagram of the working principle of a follow-up hydraulic muffler.

\section{Static Characteristic Analysis of Follow-up Hydraulic Muffler}

Fluid pulsation produced by hydraulic pump includes two parts: natural frequency and pulsation frequency,in which the pulsation frequency occupies the main position in fluid pulsation, so the pulsation frequency is mainly considered in the analysis of static characteristics. The section chart of the follow-up adjustment section is shown in Figure 3.

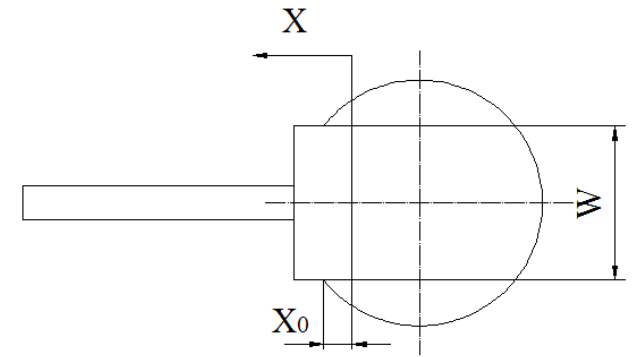

Fig. 3. Section chart of adjusting device of quality chamber with circular interface

The relationship between engine speed and pulsation frequency of hydraulic pump is ${ }^{[3]-[4]}$ :

$$
n_{d}=\frac{60}{K_{n} Z} f_{r}
$$

Pulsation frequency:

$$
f_{r}=\frac{a}{2 \pi} \sqrt{\frac{1}{\left(\frac{l_{1}}{A_{1}}+\frac{l_{2}}{A_{2}}\right) V}}
$$

The relationship between the circulation area of the quality chamber and the displacement of the sliding block is: $\quad A_{2}=A_{20}+W X$ 


$$
A_{20}=\frac{\pi}{4} d_{2}^{2}-W X_{0}-\left[\frac{1}{4} d_{2}^{2} \arcsin \left(\frac{W}{d_{2}}\right)-\frac{1}{4} W d_{2} \cos \left(\arcsin \left(\frac{W}{d_{2}}\right)\right)\right]
$$

$W$ is the width of the sliding block; $X_{0}$ is the initial occlusion length of the sliding block; $X$ is the displacement of the sliding block.According to Eq.(1)-(4),the relationship between the displacement of the sliding block and the engine speed is shown in Eq.(5); and then via Eq.(2)-(3) to work out the relationship between the displacement of the sliding block and the pulsation frequency of the follow-up hydraulic muffler is shown in Eq.(6).

$$
\begin{gathered}
X=\frac{1}{W}\left[\frac{l_{2}}{\frac{1}{V}\left(\frac{60 a}{2 \pi K_{n} Z n_{d}}\right)^{2}}-A_{20}\right] \\
f_{r}=\frac{a}{2 \pi} \sqrt{\frac{1}{\left(\frac{l_{1}}{A_{1}}+\frac{l_{2}}{A_{20}+W X}\right) V}}
\end{gathered}
$$

Moreover,this study further designed the quality chamber into square pipes, which makes the operation easier in sealing and maintenance.The design diagram is shown in Fig.4.The relationship between the displacement of the sliding block and the pulsation frequency of the follow-up hydraulic muffler is shown in Eq.(7).

$$
f_{r}=\frac{a}{2 \pi} \sqrt{\frac{1}{\left(\frac{l_{1}}{A_{1}}+\frac{l_{2}}{b^{2}-b\left(X-X_{0}\right)}\right) V}}
$$

Using (5) to derive the displacement and frequency diagram of the sliding block, as in Fig.5. Using (7) to derive the displacement of sliding block and engine speed diagram,as in Fig. 6.It can be seen from Fig. 5 and Fig. 6 that the displacement of the sliding block has a power function relationship with the frequency and the rotational speed.

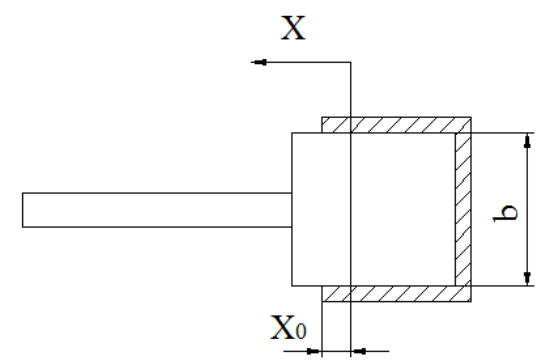

Fig.4. The section chart of the square quality chamber

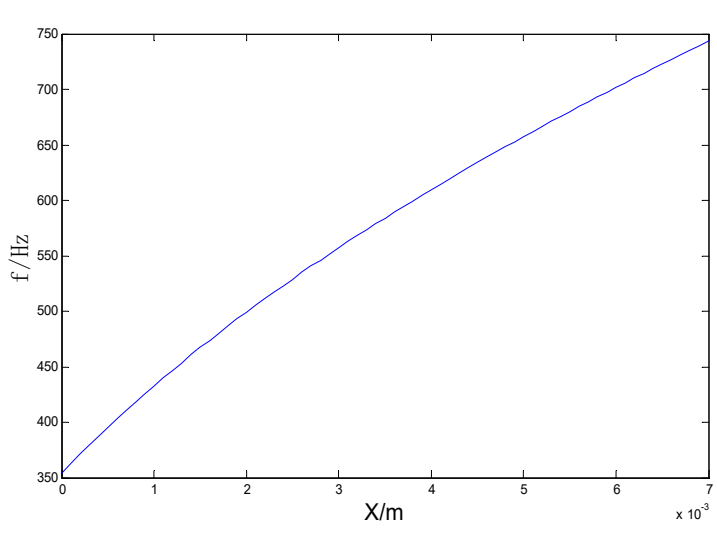

Fig.5. The diagram of the displacement of the sliding block and frequency

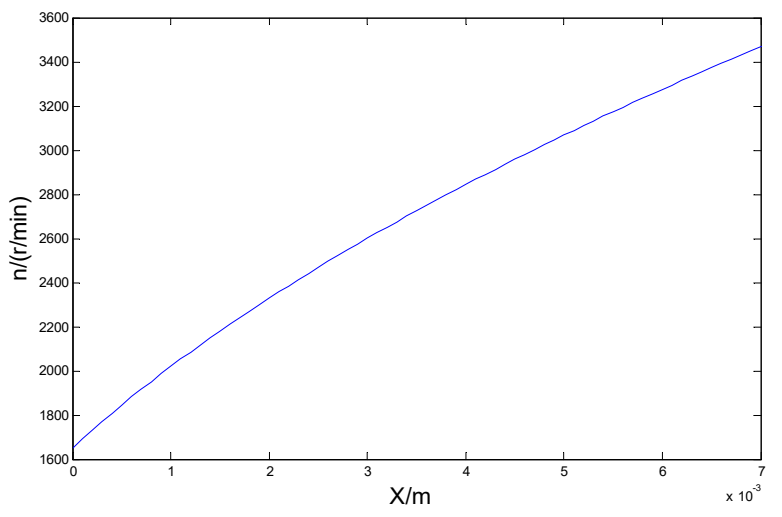

Fig.6. The diagram of the displacement of the sliding block and engine speed

\section{The Dynamic characteristics analysis of follow-up hydraulic muffler}

When the follow-up regulator is in motion, it is affected by electromagnetic force, spring force and liquid damping force. It constitutes a typical quality-elastic frame-damp system. The dynamic characteristics of the follow-up regulator have an important influence on the performance of the follow-up hydraulic muffler.

The kinetic equation of armature and sliding block

is $^{[5]-[6]}: K_{1} i=m x+K_{3} x+K_{2} x$

Set $K_{1}$ as electromagnet current / force conversion coefficient; $K_{2}$ as spring stiffness coefficient; $K_{3}$ as liquid damping coefficient; $K_{4}$ as conversion coefficient of displacement transducer, $K_{5}$ as amplification coefficient of control amplifier,when $K_{6}$ is the proportional coefficient between the input current and the desired sliding block displacement, to get the desired position of the sliding block is $r$, the transfer function of the actual sliding block position $\mathrm{X}$ is the output :

$$
\operatorname{In}, \Phi(s)=\frac{X(s)}{r(s)}=K \frac{\omega_{n}^{2}}{s^{2}+2 \xi \omega_{n} s+\omega_{n}^{2}}
$$




$$
\begin{gathered}
\omega_{n}=\sqrt{\frac{K_{2}+K_{1} K_{5} K_{6}}{m}} ; \\
\xi=\frac{K_{3}}{2 \sqrt{\left(K_{2}+K_{1} K_{4} K_{5}\right) m}} .
\end{gathered}
$$

The design requirements of the second-order dynamic transfer function of the follow-up regulator are:fast response; smooth operation; less error. So take $\xi=0.707 ; \omega_{n}=62.5 \mathrm{rad} / \mathrm{s}$;reasonably take values of coefficients $K_{1}-K_{6}$ to match $\xi$ and $\omega_{n}$,Among them, $K_{3}$ should be positive number.That is, liquid damping is positive number.So,this will satisfy the dynamic characteristics of the follow-up hydraulic muffler.Fig. 7 is the dynamic response diagram of step signal when the input displacement is $7 \mathrm{~mm}^{[7]}$. From this figure,when the stable time of the system is less than $0.15 \mathrm{~s}$, the system can meet the actual needs of the project.Of course, parameters optimization can be adjusted to make the response time more ideal.To optimize the parameters can make more ideal response time.

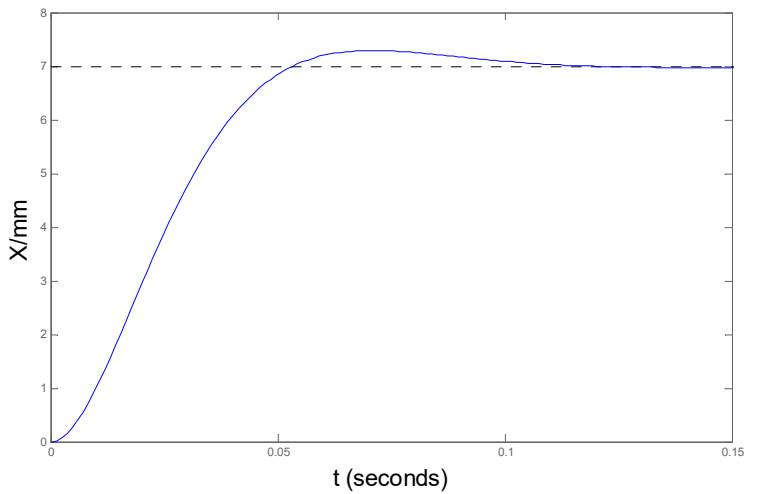

Fig.7. The step signal response diagram of the system when the input displacement is $7 \mathrm{~mm}$

\section{Conclusion}

By analyzing the static and dynamic characteristics of the follow-up hydraulic muffler based on the spring-damp regulating device, it can be concluded that this design can meet the needs of requirements in practical engineering, theoretically modeling and analyzing to make a entity model to verify, and also provide a reference for the study of hydraulic silencer.

\section{Acknowledgement}

This paper is subordinate to the Project of Scientific Research Fund of Yunnan Provincial Department of Education: The Design and Characteristics of Follow- up Hydraulic Muffler(NO:2018JS507).

\section{References}

1. Shan Changji. Machine Tool and Hydraulics, 173-175,15(2015)
2. DU Run. Machine Tool and Hydraulics,34-36,9(2010)

3. CHEN hualing.Mechanical Engineering Test Technology,30-40,(2013)

4. Bai Jing.Motion Control System,19-28,(2012)

5. Xiong Shibo. Mechanical Engineering Testing,18-36,(2015)

6. Gu Xingsheng.The Application of Modern Control Theory,114-138,(2008)

7. Huang Zhonglin. The Calculation and Simulation of Control System based on MATLAB,(2001) 